\title{
Anomalies of ac driven solitary waves with internal modes: Nonparametric resonances induced by parametric forces
}

\author{
Niurka R. Quintero* \\ Grupo de Física No Lineal, Departamento de Física Aplicada I, Universidad de Sevilla, Facultad de Informática, \\ Avenida Reina Mercedes s/n, 41012 Sevilla, Spain \\ Angel Sánchez ${ }^{\dagger}$ \\ Grupo Interdisciplinar de Sistemas Complicados (GISC), Departamento de Matemáticas, Universidad Carlos III de Madrid, \\ Avenida Universidad 30, 28911 Leganés, Madrid, Spain \\ Franz G. Mertens \\ Physikalisches Institut, Universität Bayreuth, D-95440 Bayreuth, Germany \\ (Received 30 May 2001; published 18 September 2001)
}

\begin{abstract}
We study the dynamics of kinks in the $\phi^{4}$ model subjected to a parametric ac force, both with and without damping, as a paradigm of solitary waves with internal modes. By using a collective coordinate approach, we find that the parametric force has a nonparametric effect on the kink motion. Specifically, we find that the internal mode leads to a resonance for frequencies of the parametric driving close to its own frequency, in which case the energy of the system grows as well as the width of the kink. These predictions of the collective coordinate theory are verified by numerical simulations of the full partial differential equation. We finally compare this kind of resonance with that obtained for nonparametric ac forces and conclude that the effect of ac drivings on solitary waves with internal modes is exactly the opposite of their character in the partial differential equation.
\end{abstract}

DOI: 10.1103/PhysRevE.64.046601

PACS number(s): 05.45.Yv, 02.30.Jr, 03.50.-z, 63.20.Pw

\section{INTRODUCTION}

During the last three decades of the 20th century, we have witnessed a great deal of effort devoted to the study of solitons and related nonlinear coherent excitations [1-3]. All that work notwithstanding, the analysis of systems generally regarded as paradigms of soliton phenomenology still yields unknown, surprising features. One such case is the dynamics of topological solitons or kinks in nonlinear Klein-Gordon systems subjected to ac forces. After several papers that led to contradictory conclusions during the last decade, the problem for the sine-Gordon equation was finally solved in 1998 [4]. This was the simplest possible scenario for studying the effects of ac forces on topological solitons, in so far as sineGordon kinks do not have any internal mode and behave as rigid objects [5]. However, this is a nongeneric situation, because many solitary waves do possess internal degrees of freedom [5-7], and even systems like the sine-Gordon model can acquire internal modes due to the influence of perturbations, such as discreteness [8-10]. Therefore, it was necessary to study the behavior of solitons with internal modes, and subsequently it was found that pure, nonparametric ac forces induce resonances of parametric character: If the frequency of the internal mode is denoted by $\Omega_{i}$, the resonance appears when the driving frequency is $\Omega_{i} / 2$ [11]. This anomalous behavior was shown, taking the $\phi^{4}$ equation as a typical example, to arise from the fact that the driving

\footnotetext{
*Electronic address: niurka@euler.us.es

${ }^{\dagger}$ Electronic address: anxo@math.uc3m.es

*Electronic address: franz mertens@uni-bayreuth.de
}

couples indirectly to the internal mode, which in turn leads to a parametric influence on its evolution.

In view of the above results, it is only natural to pose the following question: If nonparametric drivings act parametrically on $\phi^{4}$ kinks, what is the effect of a parametric driving? To our knowledge, this issue was first addressed in [12], although the main point of that work was to show how kinks can be effectively annihilated by a fast parametric driving, and no attention was paid to the kink dynamics. More recently, the problem was considered with both parametric and nonparametric forces acting simultaneously in the system [13], but the approach employed by those researchers was not appropriate and led to incorrect results [14]. Therefore, the dynamics of kinks in the $\phi^{4}$ model subjected to parametric drivings is largely unexplored, and this is another reason why we concern ourselves with this problem. This paper presents our conclusions on this question within the following scheme: Sec. II is devoted to our analytical approach; Sec. III contains the simulations of the full partial differential equation that verify our analytical predictions; and Sec. IV summarizes our main results.

\section{RESONANCES INDUCED BY PARAMETRIC PERIODIC FORCES}

As we are interested in the behavior of solitary waves with internal modes, we choose as our working example the $\phi^{4}$ equation, which is well known to be representative of the generic behavior of those solitary waves. Therefore, we begin by considering the perturbed problem given by

$$
\phi_{t t}-\phi_{x x}=-\frac{d U}{d \phi}-\beta \phi_{t}+f(t, \phi),
$$


where $U(\phi)=\left(1-\phi^{2}\right)^{2} / 4$ is the $\phi^{4}$ potential, $f(t, \phi)$ $=\epsilon \sin \left(\delta t+\delta_{0}\right) \phi$ represents the parametric ac force, $\epsilon, \delta$, and $\delta_{0}$ are the amplitude, frequency, and phase of the external periodic force, respectively, and $\beta$ is the damping coefficient.

In order to obtain analytical results, we resort to the wellknown collective coordinate method [5,15], which will give us definite predictions on the behavior of the system. However, these being approximate results, we will have to check them by direct numerical simulations of Eq. (1); those will be the subject of the following section. To apply the collective coordinate approach to our problem, we choose to employ the so-called generalized traveling wave ansatz [16] or, equivalently, to use the variation of the momentum and the energy (the equivalence of both techniques is proven in the second paper in [11]). These two quantities are given by

$$
\begin{gathered}
P(t) \equiv-\int_{-\infty}^{+\infty} d x \phi_{x} \phi_{t}, \\
E(t) \equiv \int_{-\infty}^{+\infty} d x\left\{\frac{1}{2} \phi_{t}^{2}+\frac{1}{2} \phi_{x}^{2}+U(\phi)\right\} .
\end{gathered}
$$

In order to evaluate those quantities, we consider the Rice ansatz [17] and assume that

$$
\phi(x, t)=\phi_{0}\left[\frac{x-X(t)}{l(t)}\right],
$$

where $\phi_{0}(x)$ is the static kink solution of the unperturbed $\phi^{4}$ equation, i.e., of Eq. (1) with $\beta=\epsilon=0$ zero, centered at $X(t)$, and with width $l(t)$. With this choice, and following the standard procedure $[5,15]$, we find that the evolution of the momentum $P(t) \equiv M_{0} l_{0} \dot{X} / l(t)$ and the width of the kink are given by

$$
\begin{gathered}
\frac{d P}{d t}=F^{\text {stat }}(X)-\beta P+F_{e x}, \\
\alpha M_{0} l_{0} \frac{\ddot{l}}{l}+\frac{P^{2}}{M_{0} l_{0}}=K^{i n t}(l, \dot{l}, \dot{X})-\beta \alpha M_{0} l_{0} \frac{i}{l}+K,
\end{gathered}
$$

with

$$
\begin{gathered}
F_{e x}=\int_{-\infty}^{+\infty} d x f(t, \phi) \frac{\partial \phi}{\partial X}, \quad F^{s t a t}=-\frac{\partial E}{\partial X}, \\
K=\int_{-\infty}^{+\infty} d x f(t, \phi) \frac{\partial \phi}{\partial l}, \quad K^{i n t}=-\frac{\partial E}{\partial l}, \\
E=\frac{1}{2} \frac{l_{0}}{l} M_{0} \dot{X}^{2}+\frac{1}{2} \frac{l_{0}}{l} \alpha M_{0} \dot{i}^{2}+\frac{1}{2} M_{0}\left(\frac{l_{0}}{l}+\frac{l}{l_{0}}\right),
\end{gathered}
$$

where $q=2, M_{0}=4 /\left(3 l_{0}\right)$, and $l_{0}=\sqrt{2}$ represent the topological charge, the mass, and the width of the unperturbed kink, respectively, and $\alpha=\left(\pi^{2}-6\right) / 12$ is a constant.

We now have to evaluate the above quantities. In this respect, it is interesting to compare the equations we have obtained with those found for the case of an ac nonparametric force, i.e., $f(t, \phi)=f(t)$. In that situation we obtain that $F_{e x}=-q f(t)$ and $K=0$, and hence the external force acts directly on the translational mode, whereas the internal mode is excited indirectly due to the coupling between these two modes. Conversely, as we have seen, in the parametrically driven problem, $F_{e x}$ vanishes, while $K=-\epsilon \sin \left(\delta t+\delta_{0}\right)$. Therefore, Eqs. (5) and (6) become

$$
\begin{gathered}
\frac{d P}{d t}=-\beta P(t), \\
\alpha\left[\dot{l}^{2}-2 l \ddot{l}-2 \beta l i\right]=\frac{l^{2}}{l_{0}^{2}}\left[1+\frac{P^{2}}{M_{0}^{2}}\right]-1+2 \frac{l(t)^{2}}{M_{0} l_{0}} \epsilon \sin \left(\delta t+\delta_{0}\right) .
\end{gathered}
$$

We thus see that, when introduced in a parametric manner, the ac force acts directly on the internal mode and not via the coupling between the translational and internal modes. This result shows the very different roles of the two drivings at the collective coordinate level.

In order to deal with these equations, we choose $\dot{X}(0)$ $=u(0), l(0) \equiv l_{s}=l_{0} \sqrt{1-u^{2}(0)}$, and $\dot{l}(0)$ as the initial conditions for Eqs. (10) and (11). The equation for the momentum is trivial and can be solved exactly, yielding

$$
P(t)=P(0) e^{-\beta t} .
$$

We note that for $\beta \neq 0$ the momentum of the kink goes to zero and after some transient time $t \gg 1 / \beta$ it effectively vanishes. In the same way, if we start from zero initial velocity, $P(t)=0$, i.e., the center of the kink will not move.

Let us now turn to Eq. (11). As a first step, we introduce a change of variables, proposed in [18], given by $l(t)$ $=g^{2}(t)$. This change transforms Eq. (11) into an Ermakovtype (or Pinney-type) equation [19], which reads

$$
\ddot{g}+\beta \dot{g}+\left[\left(\frac{\Omega}{2}\right)^{2}+\left(\frac{\Omega}{2 M_{0}}\right)^{2} P^{2}+\epsilon \frac{\sin \left(\delta t+\delta_{0}\right)}{2 \alpha M_{0} l_{0}}\right] g=\frac{1}{4 \alpha g^{3}},
$$

$$
g(0)=\sqrt{l_{s}} \neq 0, \quad \dot{g}(0)=\frac{\dot{l}(0)}{2 \sqrt{l}_{s}},
$$

where $\Omega=1 / \sqrt{\alpha} l_{0}=1.2452$ is equal to the Rice frequency [17] $\Omega_{R}=1 / \sqrt{\alpha} l_{s}$ in the case when the kink initially is at rest. It can be shown (see [17] and [20]) that when there is no perturbation in the system (10), (11), $l(t)$ oscillates with a frequency $\Omega_{R}$ if we start from any $l(0) \neq l_{s}$ or $l(0) \neq 0$. Therefore, $\Omega_{R}$ is the characteristic frequency of Eq. (11) and, since it agrees within $1.7 \%$ with $\Omega_{i}=\sqrt{3 / 2}=1.2247$, we expect that if we find a resonance related to $\Omega_{R}$ in Eq. (13) [or Eq. (11)] we should find a similar phenomenon in the full system Eq. (1), associated with the frequency of the internal mode $\Omega_{i}$. 
We now proceed to solve Eq. (13) analytically for the undamped case, i.e., when $\beta=0$. As we have mentioned, in this case $P(t)=P(0)$ and Eq. (13) becomes the following Pinney-type equation [19]:

$$
\ddot{g}+\left[\left(\frac{\Omega_{R}}{2}\right)^{2}+\epsilon \frac{\sin \left(\delta t+\delta_{0}\right)}{2 \alpha M_{0} l_{0}}\right] g=\frac{1}{4 \alpha g^{3}},
$$

whose solution is

$$
g(t)=\sqrt{v_{1}^{2}+\frac{1}{4 \alpha W^{2}} v_{2}^{2}},
$$

where $v_{1}(t)$ and $v_{2}(t)$ are two independent solutions of the linear part of Eq. (15) and $W=\dot{v}_{1} v_{2}-\dot{v}_{2} v_{1}$ is the Wronskian. $W(t)$ is actually a constant, and can be calculated from the initial conditions for $v_{i}(i=1,2), v_{1}(0)=\sqrt{l_{s}}, \dot{v}_{1}(0)$ $=\dot{l}(0) /\left(2 \sqrt{l_{s}}\right), v_{2}(0)=0$, and $\dot{v}_{2}(0)$ a nonzero constant.

If we denote $\tau=\left(\delta t+\delta_{0}+\pi / 2\right) / 2$, after some algebraic manipulations we arrive at the following Mathieu equation for the $v_{i}$ functions:

$$
\begin{gathered}
v_{i}^{\prime \prime}+[a-2 \theta \cos (2 \tau)] v_{i}=0, \\
a=\left(\frac{\Omega_{R}}{\delta}\right)^{2}, \quad \theta=\frac{\epsilon}{\alpha M_{0} l_{0} \delta^{2}} \equiv \frac{\epsilon l_{0}}{M_{0} \gamma_{0}^{2}}\left(\frac{\Omega_{R}}{\delta}\right)^{2},
\end{gathered}
$$

where prime denotes the derivative with respect to $\tau$, and $\gamma_{0}=1 / \sqrt{1-v(0)^{2}}$. Notice that the initial conditions, when $\tau \equiv \tau_{0}=\left(\delta_{0}+\pi / 2\right) / 2 \quad$ for $\quad v_{i}(\tau)$, become $\quad v_{1}\left(\tau_{0}\right)=\sqrt{l_{s}}$, $v_{1}^{\prime}\left(\tau_{0}\right)=\dot{l}(0) /\left(\delta \sqrt{l_{s}}\right), \quad v_{2}\left(\tau_{0}\right)=0$, and $v_{2}^{\prime}\left(\tau_{0}\right)=2 \dot{v}_{2}(0) / \delta$. The solution of Eq. (17) (see [21]) for $v_{1}(\tau)$ and $v_{2}(\tau)$ can be expressed as a linear superposition of the two Mathieu functions $\mathrm{ce}_{\nu}$ and $\mathrm{se}_{\nu}$ with a noninteger index $\nu$; i.e.,

$$
v_{i}(\tau)=A_{i} \operatorname{ce}_{\nu}(\tau, \theta)+B_{i} \operatorname{se}_{\nu}(\tau, \theta), \quad i=1,2,
$$

where

$$
A_{i} \equiv \frac{\Delta_{A_{i}}}{\Delta}, \quad B_{i} \equiv \frac{\Delta_{B_{i}}}{\Delta},
$$

and

$$
\begin{gathered}
\Delta=\mathrm{ce}_{\nu}\left(\tau_{0}, \theta\right) \mathrm{se}_{\nu}^{\prime}\left(\tau_{0}, \theta\right)-\mathrm{ce}_{\nu}^{\prime}\left(\tau_{0}, \theta\right) \operatorname{se}_{\nu}\left(\tau_{0}, \theta\right), \\
\Delta_{A_{i}}=v_{i}\left(\tau_{0}\right) \mathrm{se}_{\nu}^{\prime}\left(\tau_{0}, \theta\right)-v_{i}^{\prime}\left(\tau_{0}\right) \mathrm{se}_{\nu}\left(\tau_{0}, \theta\right), \\
\Delta_{B_{i}}=v_{i}^{\prime}\left(\tau_{0}\right) \mathrm{ce}_{\nu}\left(\tau_{0}, \theta\right)-v_{i}\left(\tau_{0}\right) \mathrm{ce}_{\nu}^{\prime}\left(\tau_{0}, \theta\right),
\end{gathered}
$$

with the constraint (characteristic curve for Mathieu functions)

$$
a=\nu^{2}+\frac{1}{2\left(\nu^{2}-1\right)} \theta^{2}+O\left(\theta^{4}\right) .
$$

From Eqs. (16), (18), and (19), and taking into account that $\tau=\left(\delta t+\delta_{0}+\pi / 2\right) / 2$, we obtain that the kink width $l(t)$ is given by

$$
l(t)=g^{2}=v_{1}^{2}(t)+\frac{1}{4 \alpha W^{2}} v_{2}^{2}(t),
$$

where

$$
\begin{gathered}
v_{i}(t)=A_{i} \operatorname{ce}_{\nu}\left(\delta t / 2+\delta_{0} / 2+\pi / 4, \theta\right) \\
+B_{i} \mathrm{se}_{\nu}\left(\delta t / 2+\delta_{0} / 2+\pi / 4, \theta\right), \quad i=1,2, \\
W=-\sqrt{l_{s}} \dot{v}_{2}(0),
\end{gathered}
$$

and the characteristic curve Eq. (20) for our initial parameters can be written up to order $\epsilon^{2}$ as

$$
\delta=\frac{\Omega_{R}}{\nu}\left(1-\frac{\nu^{2} l_{0}^{2}}{4\left(\nu^{2}-1\right) M_{0}^{2} \gamma_{0}^{4}} \epsilon^{2}\right)+O\left(\epsilon^{4}\right) .
$$

Notice that when $\nu=m+p / s$ is rational, with $m$ an integer number and $p / s$ a rational fraction $(0<p / s<1), v_{1}(t)$ and $v_{2}(t)$ are $2 \pi s$-periodic functions, if $p$ is odd, and $\pi s$-periodic functions, if $p$ is even, whereas for irrational $\nu$ both functions will be nonperiodic although bounded [21]. For instance, if we take in Eq. (11), $\epsilon=0.01, \delta_{0}=\pi / 2$, $u(0)=0, l(0)=l_{s}$ and $\delta=0.94$, we can see in Fig. 1 that $l(t)$ is a bounded function. Moreover, taking the Mathieu functions that appear in Eq. (22) up to terms of order $\theta$ and substituting the approximate expression of Eq. (22) in Eq. (21) one can show that the resulting expression for $l(t)$ involves basically the frequencies $\Omega_{R}$ and $\delta$ (see Fig. 1). We have also plotted in Fig. 1 the evolution of the energy, Eq. (9), taking $\delta$ not close to $\Omega_{R}$. For this choice of parameters, $E(t)$ is also a bounded function and its spectrum for the above parameters chiefly involves three frequencies: $\left|\delta-\Omega_{R}\right|, 2 \delta$, and $\delta+\Omega_{R}$.

As we have shown, Eq. (24) represents the characteristic curve corresponding to stable (either periodic or bounded) solutions of the Mathieu equation (17). However, if we try to find a periodic solution when $\delta \approx \Omega_{R}$, we obtain that the integer Mathieu functions $\mathrm{se}_{1}$ and $\mathrm{ce}_{1}$ are two independent solutions of two different Mathieu equations since they are related to different characteristic curves:

$$
\begin{aligned}
& a=1-\theta-\frac{\theta^{2}}{8}+\frac{\theta^{3}}{64}-\frac{\theta^{4}}{1536}+\cdots, \\
& a=1+\theta-\frac{\theta^{2}}{8}-\frac{\theta^{3}}{64}-\frac{\theta^{4}}{1536}+\cdots,
\end{aligned}
$$

respectively. These characteristic curves separate the unstable and stable regions of Eq. (17). In the unstable regions the solution of this equation, as well as $l(t)$ and $E(t)$, grows with $t$ (see Fig. 2). This is hence a hint of the existence of a resonance phenomenon, and, as we already pointed out, we expect that it will manifest itself in the full partial differential equation in an increment of the width and the energy of the 

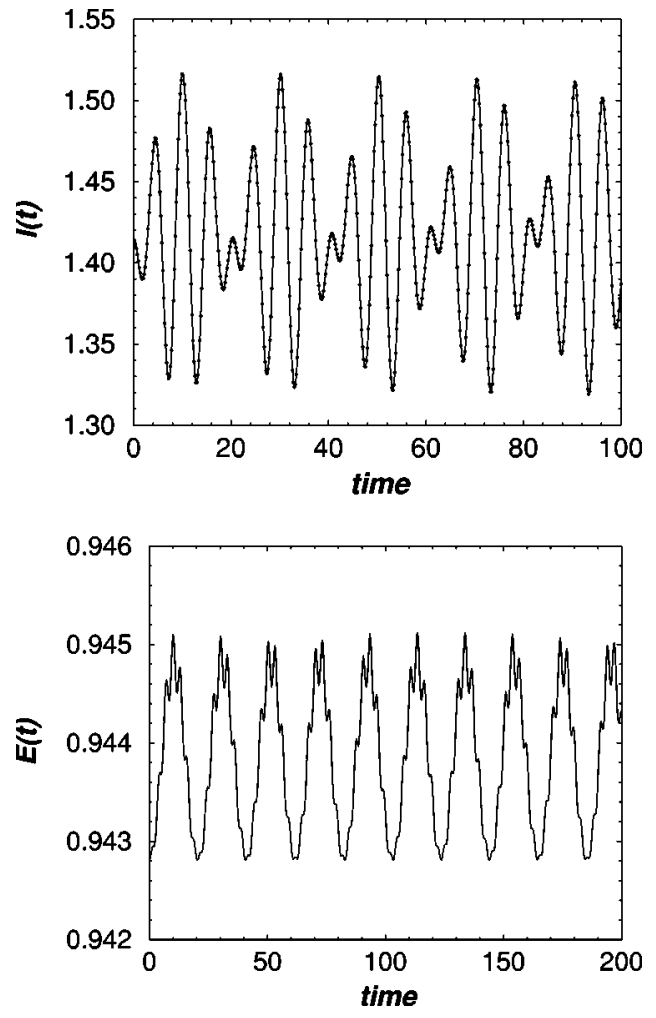

FIG. 1. Collective coordinates: Evolution of $l(t)$ and $E(t)$ when $\delta=0.94$ for the undamped case, $\beta=0$. Points: Analytical solution [see Eqs. (18)-(23)]. Solid lines: Numerical integration of Eq. (11) for $\beta=0, \epsilon=0.01, \delta_{0}=\pi / 2, u(0)=0, X(0)=0$. The DFT (not shown) of $l(t)$ yields two frequencies: $\omega_{1}=0.9434 \approx \delta$ and $\omega_{2}$ $=1.25789 \approx \Omega_{R}$, whereas for $E(t)$ it gives three frequencies: $\omega_{1}$ $=0.3144 \approx\left|\delta-\Omega_{R}\right|=0.3052, \quad \omega_{2}=1.8868 \approx 2 \delta=1.88$, and $\omega_{3}$ $=2.20131 \approx \delta+\Omega_{R}=2.1852$. The maximum of the discrete Fourier transform appears at $\omega_{1}$.

kink, when $\delta$ is close to $\Omega_{i}$. This is the aim of the next section, in which we will check by computing the solution of Eq. (1) whether there is some resonance at $\delta \approx \Omega_{i}$. In addition, we will analyze the effect of damping in our resonance picture numerically, as we have not been able to obtain an analytical approximate result in that case.

\section{SIMULATION RESULTS}

In this section we carry out numerical simulations of Eq. (1) by using the Strauss-Vázquez scheme [22], taking the length of the system $L=100, \Delta t=0.01, \Delta x=0.1$, the final time $t_{f}=200$, and free boundary conditions. We also fix the amplitude $\epsilon=0.01$ and the phase $\delta_{0}=\pi / 2$ of the ac force.

In order to show the evolution of $l(t)$ and $E(t)$ in the partial differential equation (PDE) (1) we distinguish two cases: In the first case we choose $\delta$ far away from $\Omega_{i}$, whereas in the second one $\delta \approx \Omega_{i}$. In Fig. 3 we plot the width and the energy of the kink as functions of $t$ for $\delta$ $=0.94$, i.e., the parameters of the ac force are the same as in Fig. 1. If we compare Figs. 1 and 3 we see that $l(t)$ and $E(t)$ have the same behavior both on the collective coordinate level and in the full system Eq. (1). Naturally, while in the
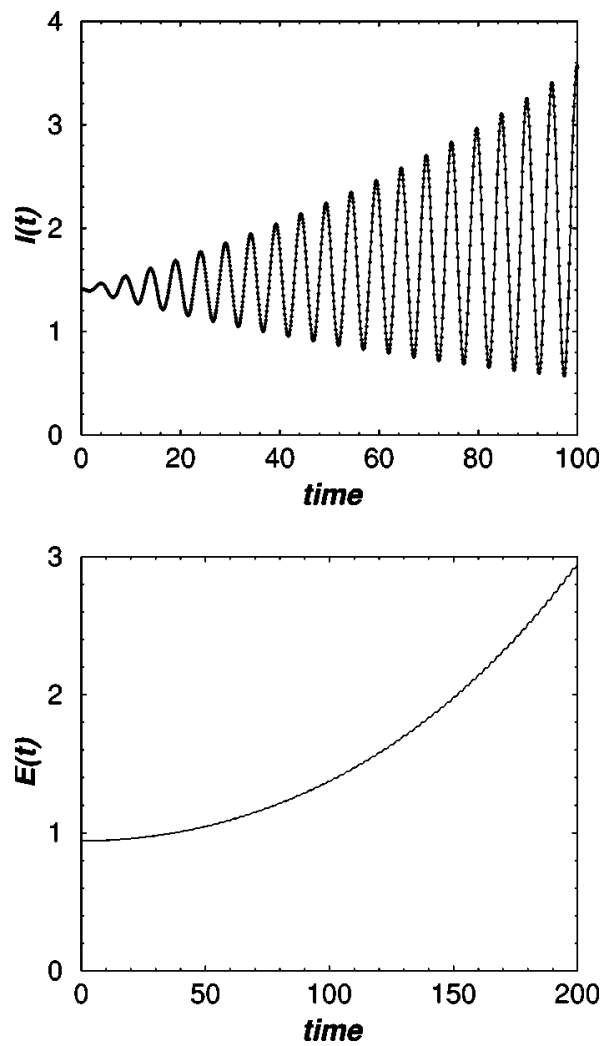

FIG. 2. Collective coordinates: Evolution of $l(t)$ and $E(t)$ when $\delta=1.24 \approx \Omega_{R}$ for the undamped case, $\beta=0$. Points: Analytical solution [see Eqs. (19)-(23)]. Solid lines: Numerical integration of Eq. (11). Parameters are as in the previous figure.

spectrum of $l(t)$ obtained by the collective coordinate approach there appear the frequencies $\delta$ and $\Omega_{R}$, in the DFT of $l(t)$ for the PDE we obtain $\delta$ and $\Omega_{i}$, as expected. However, in the Fourier transform of $E(t)$ for the full system we find not only frequencies that involve $\delta$ and $\Omega_{i}$ (see Fig. 3), but also an additional frequency $\omega_{4}=0.4715 \approx \omega_{0}-\delta$ related to the frequency of the lowest phonon $\left(\omega_{0}=\sqrt{2}\right)$ of the $\phi^{4}$ system. This means that the internal mode and the phonons can both appear when the $\phi^{4}$ system is driven parametrically with an ac force, but since $\Omega_{i}$ and $\omega_{0}$ are separated we expect to be able to excite the internal mode to a larger extent than phonon modes if the frequency of the ac force is closer to $\Omega_{i}$ than to $\omega_{0}$.

Moving now to the second case, i.e., drivings with frequency $\delta$ close to $\Omega_{i}$, Fig. 4 shows that in this situation the width of the kink oscillates with a large amplitude while the energy grows, although not monotonically. It is important to stress at this point that, whereas the collective coordinate analysis predicts that $l(t)$ and $E(t)$ should increase indefinitely at resonance, this is not the case in the original system. The reason is that, in our perturbative method, we have not taken into account the phonons that are present in the system, and therefore part of the energy input from the ac force goes to the phonons. In our simulations we have also seen that the kink does not move, even if we are at resonance, in agreement with our collective coordinate theory.

In order to present the resonance in a more evident man- 

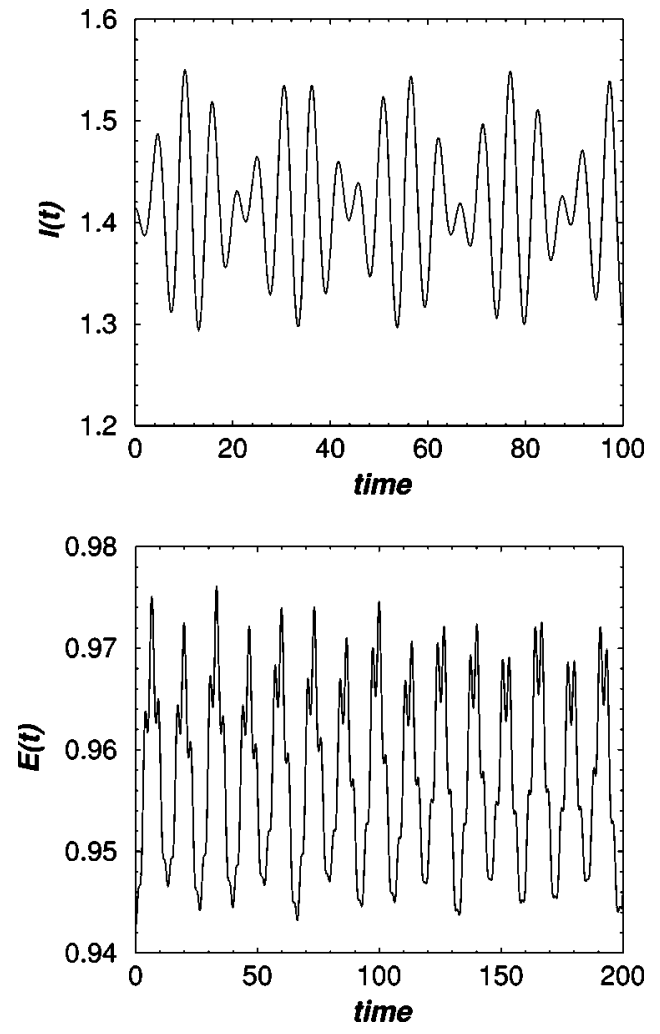

FIG. 3. Evolution of $l(t)$ and $E(t)$ as obtained from numerical simulations of Eq. (1), when $\delta=0.94$ with $\epsilon=0.01, \delta_{0}=\pi / 2$, $u(0)=0, X(0)=0$. The DFT (not shown) of $l(t)$ yields two frequencies: $\omega_{1}=0.9429 \approx \delta$ and $\omega_{2}=1.2258 \approx \Omega_{i}$, whereas for $E(t)$ it gives four frequencies: $\omega_{1}=0.2887 \approx\left|\delta-\Omega_{i}\right|=0.2847, \omega_{2}$ $=1.8859 \approx 2 \delta=1.88, \quad \omega_{3}=2.3574 \approx \delta+\Omega_{i}=2.1647, \quad$ and $\omega_{4}$ $=0.4715 \approx \omega_{0}-\delta=0.4749$.

ner, we have plotted in Fig. 5 the values of the mean normalized width and the mean energy as functions of $\delta$. We compute these magnitudes as time averages of $l(t) / l_{0}$ and $E(t)$ for times $100 \leqslant t \leqslant 200$, so as to avoid possible transient effects (which are mostly relevant in the damped case discussed below). We see that on the collective coordinate level $\left\langle l(t) / l_{0}\right\rangle$ and $\langle E(t)\rangle$ have a maximum at $\delta=\Omega_{R}$; correspondingly, in the original system Eq. (1), the resonance takes place at $\delta=1.18$, close to $\Omega_{i}=1.2247$. We have also found that for $1.3<\delta<\omega_{0}$ (not shown), the energy is an increasing function of $\delta$ whereas the width of the kink is essentically constant. This implies that the increment of the energy is due to excitation of the phonons, which becomes more efficient as $\delta \rightarrow \omega_{0}$, and is not related to the internal mode.

Coming now to the damped case, as we mentioned in the preceding section, we have not obtained an analytical result. For this reason, in this section we carry out numerical simulations of Eq. (1) and also integrate numerically the collective coordinate prediction Eq. (11) when $\beta \neq 0$. In Fig. 6 we plot the evolution of the energy at resonance for $\beta=0.05$, as given by the collective coordinates and as obtained from the numerical simulations of Eq. (1). For each case, we have chosen a frequency near to $\Omega_{R}$ (for the collective coordinates) and $\Omega_{i}$ [for Eq. (1)]. To complete the study of the
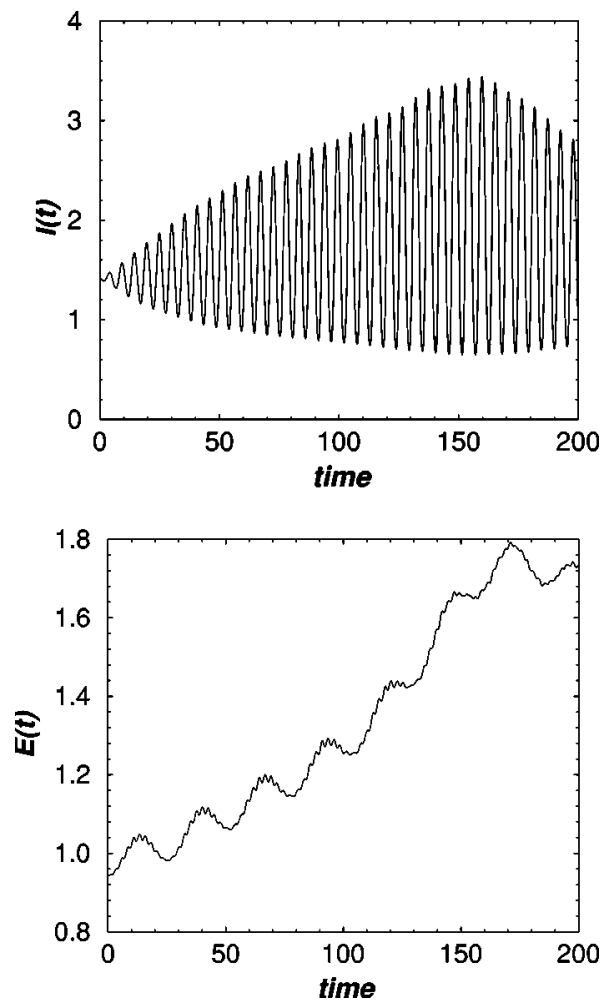

FIG. 4. Evolution of $l(t)$ and $E(t)$ as obtained from numerical simulations of Eq. (1), when $\delta=1.176$. Other parameters are the same as in the previous figure.
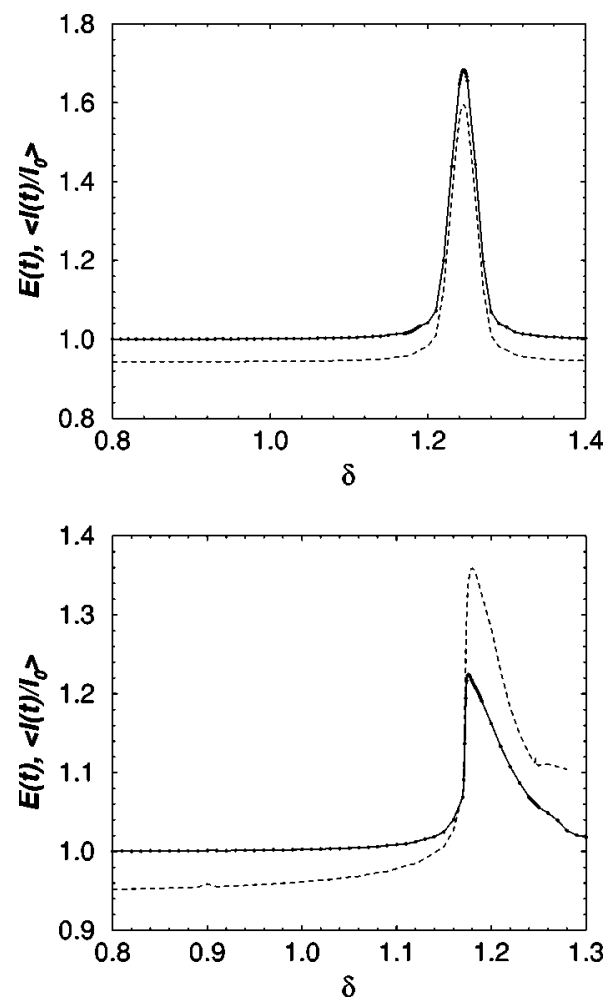

FIG. 5. $\left\langle l(t) / l_{0}\right\rangle$ (points connected by solid line) and $\langle E(t)\rangle$ (dashed line) vs $\delta$. Upper panel: Collective coordinate approach. The resonance is at $\Omega_{R}=1.2452$. Lower panel: Results from the full system Eq. (1). The resonance is at $\delta=1.18$, close to $\Omega_{i}=1.2247$. 

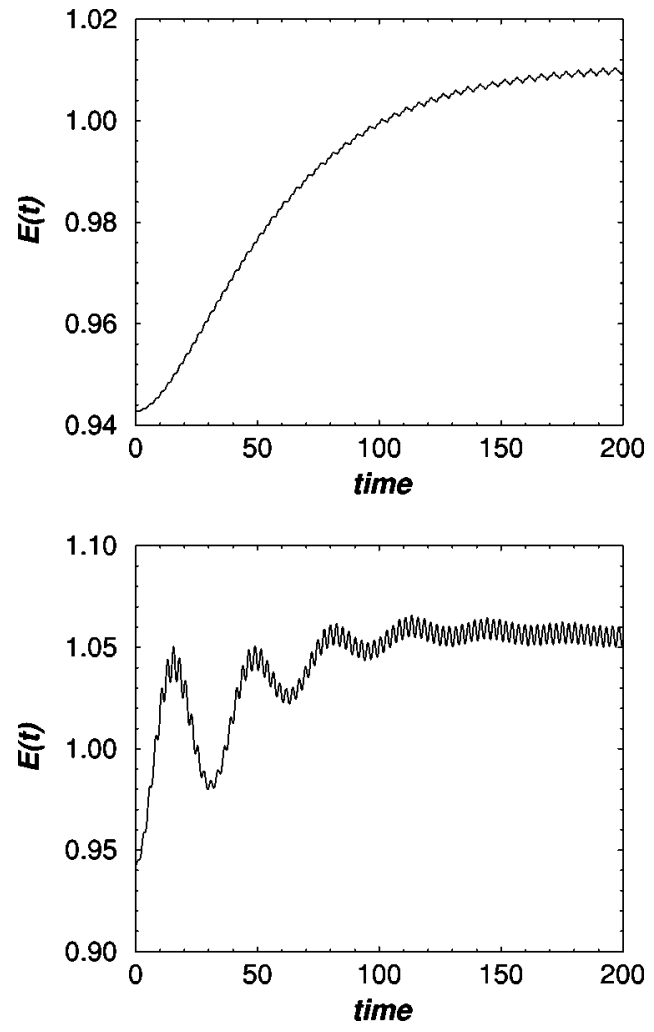

FIG. 6. Damped case, $\beta=0.05$ : Evolution of the energy according to the collective coordinate approach, obtained by numerical integration of Eq. (11), and from the full system, by numerical simulation of Eq. (1), when $\delta=1.245 \approx \Omega_{R}$ and $\delta=1.22 \approx \Omega_{i}$, respectively.

damped case, we present the result for the resonance as we did for the undamped case, by plotting the mean width and energy vs $\delta$ for $\beta=0.05$ (see Fig. 7). We can observe that, at resonance, these functions also have maxima, although in the presence of damping they are less peaked than in the undamped case: $\left\langle l(t) / l_{0}\right\rangle$ increases by around $7 \%$ and the energy by around $10 \%$. Interestingly, the resonance for the full system is now much closer to the internal mode than in the undamped case. We believe that the fact that the damping suppresses the phonons quite effectively is the reason why the damped system follows more closely the collective coordinate prediction, which indeed neglects all phonon contributions.

\section{CONCLUSIONS}

In the present paper we have studed the effect of a parametric ac force on the dynamics of $\phi^{4}$ kinks with and without damping. By using a collective coordinate approach, we have found resonances related to the excitation of the internal mode when the frequency of the ac force is close to the Rice frequency $\Omega_{R} \approx \Omega_{i}$. We have verified this prediction numerically by computing the solution of Eq. (1), its corresponding energy, and the width of the kink. When this kind of resonance occurs, the energy and the width of the system increase, while for other values of the frequency (except for smaller, secondary resonances) they are bounded (in some
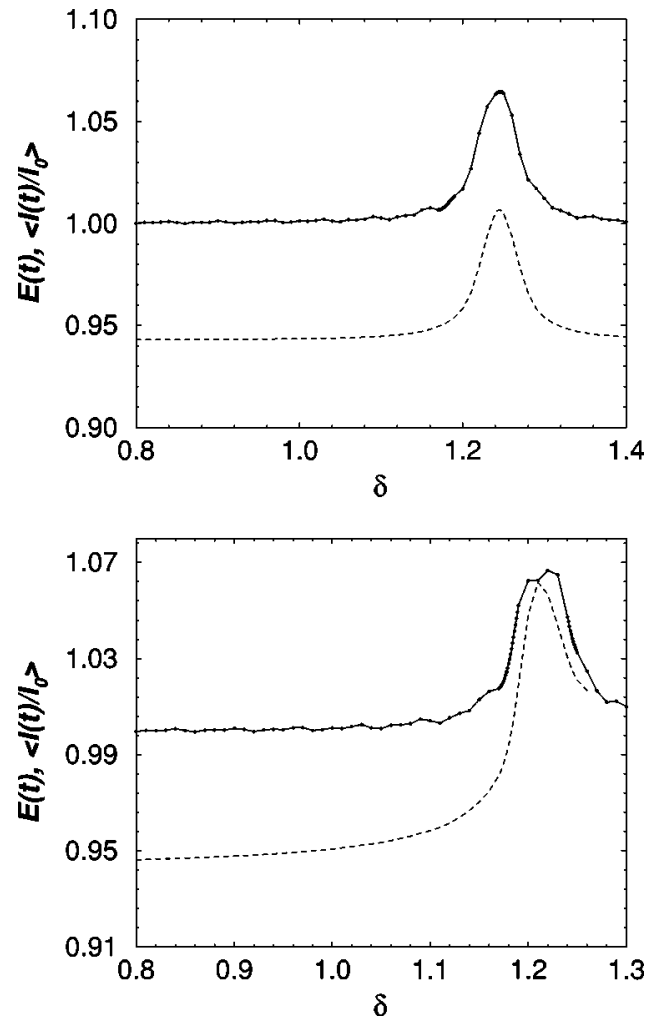

FIG. 7. Damped case, $\beta=0.05:\left\langle l(t) / l_{0}\right\rangle$ (points connected by solid line) and $\langle E(t)\rangle$ (dashed line) vs $\delta$. Upper panel: Collective coordinate approach. The width and the energy take their maximum values at $\delta=1.246$ and $\delta=1.245$, respectively. Lower panel: Results from the full system Eq. (1). The effective width has its maximum at $\delta=1.22$ and the maximum of the energy occurs at $\delta$ $=1.21$, both of them close to $\Omega_{i}=1.2247$.

cases periodic) functions. Concerning the mobility of the kink, it is very important to point out, at least for the time considered here, that all the input energy goes to the internal and phonon modes and for this reason the kink does not move (either near or away from the resonance).

Although this work has focused on the $\phi^{4}$ kink, experience shows that it is very likely that similar phenomenologies will be observed in the dynamics of other solitary waves with internal modes. In this respect, it is important to compare the above results to those obtained for nonparametric ac drivings [11] in order to give a thorough, coherent picture of the problem. The collective coordinate analysis, confirmed by direct numerical simulations of the full system, shows that when we drive the $\phi^{4}$ system parametrically the force acts directly and nonparametrically on the width of the kink, i.e., the internal mode is excited and the phenomenon of resonance takes place and can be observed by monitoring $l(t)$ and $E(t)$. On the contrary, non-parametric drivings affect the internal mode only indirectly through the coupling to the translation mode, which in turn leads to a parametric coupling. We thus see that the internal mode dynamics is responsible for a highly nontrivial behavior: nonparametric drivings on the full system induce parametric resonances in the kink dynamics, and vice versa. As a final remark, we want to point out that our results indicate a way of exciting 
internal modes without affecting the translation mode, which can mask the effects one is interested in. As we have seen, the parametric ac force affects only the internal mode, and the kink remains at its original position (or moving uniformly with the same speed if nonzero) at all times. This is not possible with a nonparametric driving and might be a mechanism of interest in situations where the role of internal modes must be elucidated.

\section{ACKNOWLEDGMENTS}

Work at Sevilla was supported by the EU Grant No. LOCNET HPRN-CT-1999-00163. Work at Leganés was supported by DGI of MCyT (Spain) through Grant No. BFM2000-0006. Travel between Spain and Bayreuth was supported by the Emil-Warburg-Foundation of Universität Bayreuth.
[1] M. Remoissenet, Waves Called Solitons (Springer, Berlin, 1996).

[2] A.C. Scott, Nonlinear Science (Oxford University, Oxford, 1999).

[3] Nonlinear Science at the Dawn of the 21st Century, edited by P.L. Christiansen, M.P. Sorensen, and A.C. Scott (Springer, Berlin, 2000).

[4] N.R. Quintero and A. Sánchez, Phys. Lett. A 247, 161 (1998); Eur. Phys. J. B 6, 133 (1998).

[5] Yu.S. Kivshar and B.A. Malomed, Rev. Mod. Phys. 61, 763 (1989).

[6] M. Peyrard and M. Remoissenet, Phys. Rev. B 26, 2886 (1982).

[7] M. Peyrard and D.K. Campbell, Physica D 9, 33 (1983); D.K. Campbell, J.F. Schonfeld, and C.A. Wingate, ibid. 9, 1 (1983); D.K. Campbell, M. Peyrard, and P. Sodano, ibid. 19, 165 (1986).

[8] Yu.S. Kivshar, D.E. Pelinovsky, T. Cretegny, and M. Peyrard, Phys. Rev. Lett. 80, 5032 (1998).

[9] P.G. Kevrekidis and C.K.R.T. Jones, Phys. Rev. E 61, 3114 (2000)

[10] T. Kapitula, P.G. Kevrekidis, and C.K.R.T. Jones, Phys. Rev.
E 63, 036602 (2001).

[11] N.R. Quintero, A. Sánchez, and F.G. Mertens, Phys. Rev. Lett. 84, 871 (2000); Phys. Rev. E 62, 5695 (2000).

[12] Yu.S. Kivshar, A. Sánchez, and L. Vázquez, Phys. Rev. A 45, 1207 (1992).

[13] A.L. Sukstanskii and K.I. Primak, Phys. Rev. Lett. 75, 3029 (1995).

[14] Yu.S. Kivshar and A. Sánchez, Phys. Rev. Lett. 77, 582 (1996).

[15] A. Sánchez and A.R. Bishop, SIAM Rev. 40, 579 (1998).

[16] F.G. Mertens, H.J. Schnitzer, and A.R. Bishop, Phys. Rev. B 56, 2510 (1997).

[17] M.J. Rice, Phys. Rev. B 28, 3587 (1983).

[18] E. Majerníková, Yu.B. Gaididei, and O.M. Braun, Phys. Rev. E 52, 1241 (1995).

[19] E. Pinney, Proc. Am. Math. Soc. 1, 681 (1950).

[20] N.R. Quintero, A. Sánchez, and Franz Mertens, Eur. Phys. J. B 19, 107 (2001).

[21] N.W. McLachlan, Theory and Application of Mathieu Functions (Dover, New York, 1964).

[22] W.A. Strauss and L. Vázquez, J. Comput. Phys. 28, 271 (1978). 